\title{
Preparation and immunogenicity of a purified influenza virus haemagglutinin and neuraminidase subunit vaccine
}

\author{
W. G. LAVER \\ Ph.D. \\ R. G. WEBSTER* \\ Ph.D.

\begin{abstract}
Department of Microbiology, John Curtin School of Medical Research, Australian National University, Canberra, A.C.T. 2601 Australia, and * St Jude Children's Research Hospital, Memphis, Tennessee 38101, U.S.A.
\end{abstract}

\begin{abstract}
Summary
A vaccine was prepared containing the haemagglutirin and neuraminidase subunits from the $A / P$ Port Chalmers. $1 / 73\left(\mathrm{H}_{3} \mathrm{~N}_{2}\right)$ strain of influenza virus. The virus particles were disrupted with ammonium deoxycholate and the matrix protein, which was insoluble in this detergent, was removed by centrifugation. Following removal of deoxycholate, the haemagglutinin and neuraminidase subunits aggregated by their hydrophobic ends, forming mixed clusters. These were then freed from nucleocapsids by electrophoresis.

The haemagglutinin and neuraminidase subunits were as effective as intact inactivated virus (at equivalent concentration) in eliciting a late primary antibody response when injected in saline into rabbits.

In humans also, the subunits were as immunogenic as intact inactivated virus particles at equivalent concentration. Many people, however, responded only to the 'common' antigenic determinant(s) on the haemagglutinin subunit of Port Chalmers/73 virus and did not respond at all to the 'specific' determinant(s). Sera from these people contained antibodies which reacted equally as well with Hong Kong/68 virus as with the Port Chalmers/73 strain and none which reacted exclusively with the latter virus.
\end{abstract}

\section{Introduction}

Inactivated influenza virus vaccines which contain intact virus particles may produce toxic reactions when inoculated parenterally into man (Salk, 1948; Quilligan, Francis and Minuse, 1949). This adverse toxicity can be abolished by disrupting the virus particles with ether or detergents, but such disrupted virus vaccines contain (in addition to the two surface antigens) internal components of the virus which, it is thought, play no role in the stimulation of neutralizing antibody and are therefore undesirable components of the vaccine.

We are now describing the preparation of purified haemagglutinin and neuraminidase subunits by a relatively simple method, readily adaptable to largescale production, using reagents already accepted for administration to man. The immunogenicity in rabbits of the haemagglutinin (HA) and neuraminidase (NA) is described and some unexpected findings regarding their immunogenicity in man.

Materials and methods

Virus

The Port Chalmers variant of Hong Kong influenza virus (A/Port Chalmers/1/73; $\mathrm{H}_{3} \mathrm{~N}_{2}$ ) was grown in the allantoic sac of 11-day-old embryonic chicken eggs. The virus particles were purified from the infected allantoic fluid by adsorption and elution on washed chicken erythrocytes and were sedimented from the eluates by centrifugation. The virus particles were purified further by sedimentation through a sucrose gradient $(10-40 \%$ sucrose in $0.15 \mathrm{~mol} / \mathrm{l}$ $\mathrm{NaCl}, 0.01 \mathrm{~mol} / \mathrm{l}$ sodium phosphate $\mathrm{pH} \mathrm{7.2)}$ as described (Laver, 1969), and finally suspended in $0.15 \mathrm{~mol} / \mathrm{l} \mathrm{NaCl}$ or a solution containing $2 \%$ ammonium chloride and $0.01 \mathrm{~mol} / 1$ diammonium hydrogen phosphate ( $\mathrm{pH}$ about $7 \cdot 5$ ).

\section{Disruption of virus and separation of the $M$ (matrix or} membrane) protein

The purified $\mathrm{A} / \mathrm{Port}$ Chalmers/1/73 virus particles, suspended in $2 \% \mathrm{NH}_{4} \mathrm{Cl}, 0.01 \mathrm{~mol} / 1\left(\mathrm{NH}_{4}\right)_{2} \mathrm{HPO}_{4}$ at a concentration of approximately $10^{6}$ haemagglutinating units (HAu)/ml were disrupted with ammonium deoxycholate. Sodium ions needed to be kept out, otherwise gelling occurred. Ammonium deoxycholate, $5 \%$, was added to the suspension of virus in ammonium chloride-diammonium hydrogen phosphate, with stirring at $20^{\circ} \mathrm{C}$, to give a final ammonium deoxycholate concentration of $0.2 \%$. Sometimes transient clearing of the opalescent virus suspension occurred during the addition of the ammonium deoxycholate, but usually there was no noticeable change in the opacity of the virus suspension after addition of this detergent (virus disrupted with sodium deoxycholate, on the other hand, is almost water-clear).

The virus particles disrupted with ammonium deoxycholate were allowed to stand for about $5 \mathrm{hr}$ at room temperature $\left(20^{\circ} \mathrm{C}\right)$. During this time the 
membrane (M) protein flocculated and this was accompanied by a change in opalescence. The flocculated $\mathbf{M}$ protein was then removed by low speed centrifugation $\left(10,000 \mathrm{~g}\right.$ for $20 \mathrm{~min}$ at $\left.20^{\circ} \mathrm{C}\right)$ and the supernatant was dialysed to remove the ammonium deoxycholate.

\section{Removal of ammonium deoxycholate}

The supernatant after removal of the $\mathbf{M}$ protein was dialysed against $2 \% \mathrm{NH}_{4} \mathrm{Cl}$ containing 0.01 $\mathrm{mol} / 1\left(\mathrm{NH}_{4}\right)_{2} \mathrm{HPO}_{4}$ for $48 \mathrm{hr}$ at $4^{\circ} \mathrm{C}$. It was then dialysed against phosphate buffered saline (PBS) $(0.15 \mathrm{~mol} / 1 \mathrm{NaCl}, 0.01 \mathrm{~mol} / 1$ sodium phosphate buffer $\mathrm{pH} \mathrm{7 \cdot 2)}$ for a further $24 \mathrm{hr}$. Under these conditions gel formation did not occur and the deoxycholate content was reduced to undetectable levels (Szalkowski and Mader, 1952).

\section{Sedimentation through sucrose}

The dialysed material was centrifuged at low speed $(10,000 \mathrm{~g}, 10 \mathrm{~min})$ and the small amount of precipitate was discarded. The supernatant was then layered onto an equal volume of $35 \%(w / v)$ sucrose in PBS and centrifuged at $200,000 \mathrm{~g}$ for at least $6 \mathrm{hr}$. The opalescent supernatant was discarded and the clear pellet was resuspended by sonication in PBS to give an almost water-clear solution with a faint blue opalescence. This material contained the haemagglutinin and neuraminidase subunits of the virus together with the nucleocapsids and was stored frozen at $-20^{\circ} \mathrm{C}$, or at $4^{\circ} \mathrm{C}$ with sodium azide added to prevent bacterial growth. If the preparation was to be used for inoculation into man, it was treated with formaldehyde and thiomersal and safety-tested by standard methods.

\section{Separation of nucleocapsids from haemagglutinin and neuraminidase}

Nucleocapsids were separated from haemagglutinin and neuraminidase by electrophoresis on cellulose acetate or paper strips. This was done as described previously (Laver, 1964) except that the buffer did not contain any detergent.

\section{Immunization procedures}

Young adult New Zealand white rabbits were injected i.m. with varying doses of haemagglutinin plus neuraminidase (with or without nucleocapsids) and equivalent doses of intact Port Chalmers virus. All antigens were injected in saline without adjuvant. The rabbits were reinjected after 30 days and were bled at 7 day intervals and after the second inoculation.

Adult volunteers were immunized with A/Port Chalmers/73 inactivated virus vaccine and with equivalent doses of Port Chalmers haemagglutinin plus neuraminidase subunit vaccines. Sera were col- lected 28 days after injection of the vaccines and $7 \stackrel{2}{7}$ and 28 days after a second dose of the same vaccine.

Results and discussion

Disruption of virus and separation of $M$ protein

A/Port Chalmers/ 73 virus particles were disrupted $\bar{C}$ with ammonium deoxycholate and the insoluble $\mathbf{M}$ 을 (matrix or membrane) protein was removed by centrifuging.

The supernatant remaining after removal of the $M$ protein was dialysed to remove ammonium de- $\vec{O}$ oxycholate and the haemagglutinin, neuraminidase and neucleocapsid antigens were sedimented through $\overrightarrow{\vec{\omega}}$ a layer of $35 \%$ sucrose. The supernatant, which was $\stackrel{\circ}{\circ}$ opalescent and probably contained the lipid of the virus, was discarded and the antigens were re- 3 . suspended in saline to give an almost water-clear of solution with a faint blue opalescence. Examination 8 of this material in the electron microscope showed $\varnothing$ that it contained small clusters of haemagglutinin and neuraminidase subunits and also free nucleo- $\omega$ capsids (Fig. 1).

Recovery of haemagglutinin activity varied be- $\rightarrow$ tween 100 and $200 \%$, but haemagglutinin activity $\subseteq$ may either increase or decrease depending on the $\vec{\Phi}$ state of aggregation of the subunits (as well as the $\overrightarrow{0}$ cells used in the haemagglutinin tests). Therefore these values give little idea of the recovery of $\mathrm{HA}$ Single-radial-immunodiffusion tests showed 68 recovery of haemagglutinin antigen.

Recovery of neuraminidase activity in the supernatant was approximately $70 \%$. Traces of neuraminidase only were found in the $\mathbf{M}$ protein precipitate, $\unrhd$ therefore it is likely that about $30 \%$ of the neur- $\overrightarrow{\overrightarrow{0}}$ aminidase was inactivated during the disruption 3 process and subsequent dialysis. Single-radialimmunodiffusion tests showed $79 \%$ recovery of neuraminidase antigen.

Separation of haemagglutinin and neuraminidase from nucleocapsids

The nucleocapsids were removed from the clusters of haemagglutinin and neuraminidase subunits by electrophoresis on cellulose acetate strips in buffer 음 which did not contain detergent. The nucleocapsids $D$ had a higher electrophoretic mobility at pH 9 than the haemagglutinin and neuraminidase subunits $\tilde{D}$ (which migrated together) and separation of the nucleocapsids from the other two antigens was obtained (Fig. 1).

Immunogenicity of the haemagglutinin and neuramini-co dase subunits in rabbits

Different doses of the haemagglutinin plus neur- $\stackrel{+}{+}$ aminidase clusters, with and without the nucleo- $T$ protein, were injected in saline intramuscularly into rabbits. Equivalent doses of intact A/Port Chalmers/ 


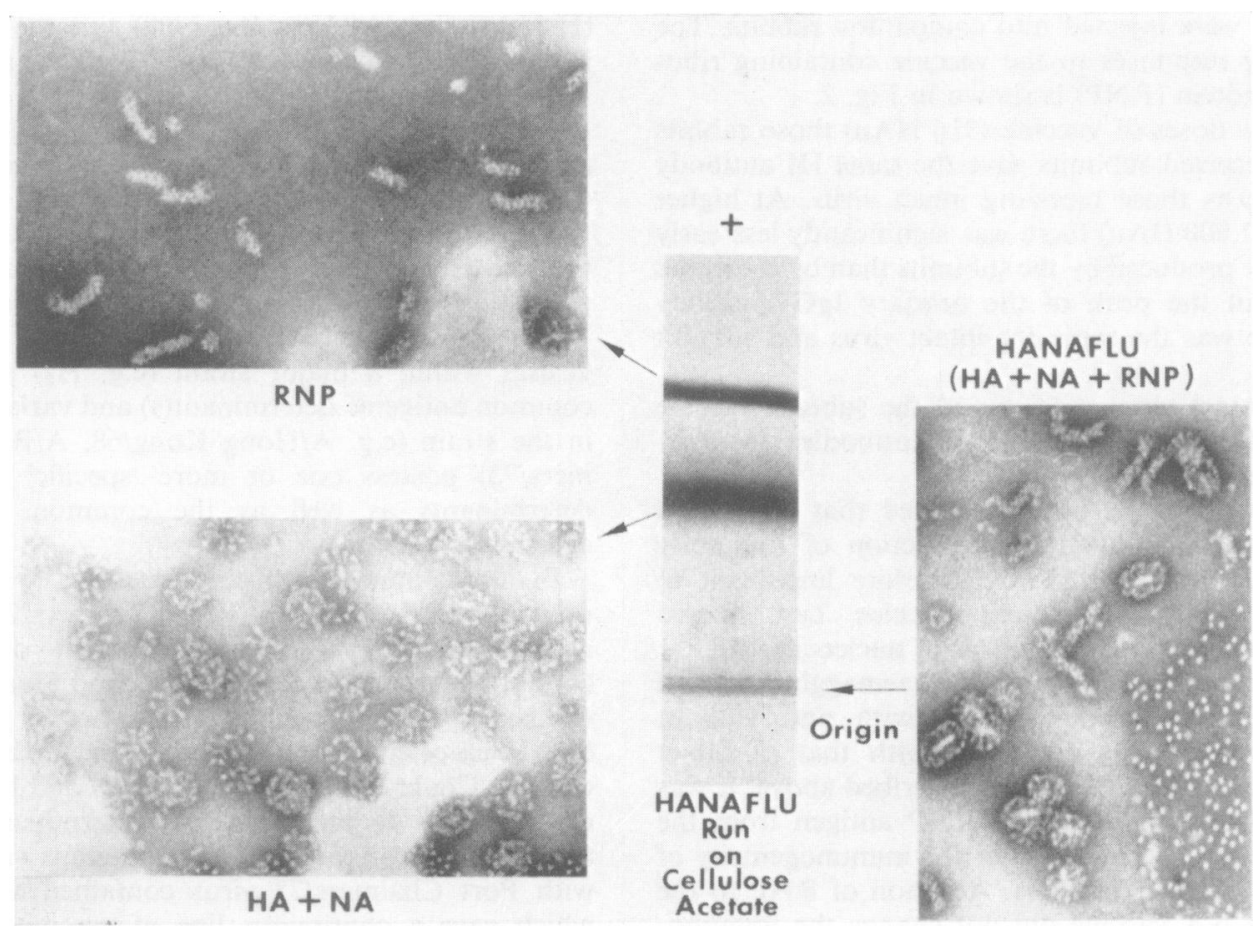

FIG. 1. Separation of nucleocapsids from the haemagglutinin and neuraminidase subunits.

The mixture of haemagglutinin, neuraminidase and nucleocapsids (termed: 'HANAFLU'), obtained from ammonium deoxycholate-disrupted A/Port Chalmers/73 virus, was subjected to electrophoresis on cellulose acetate strips. A strip, stained with Coomassie Blue, is shown. Shown also are electron micrographs $(\times 100,000)$ of the original material and the separated nucleocapsid and HA - NA fractions, eluted from the strips. The HA and NA subunits have aggregated by their hydrophobic ends in the way described by Laver and Valentine (1969). Electron micrographs were taken by Dr N. G. Wrigley.

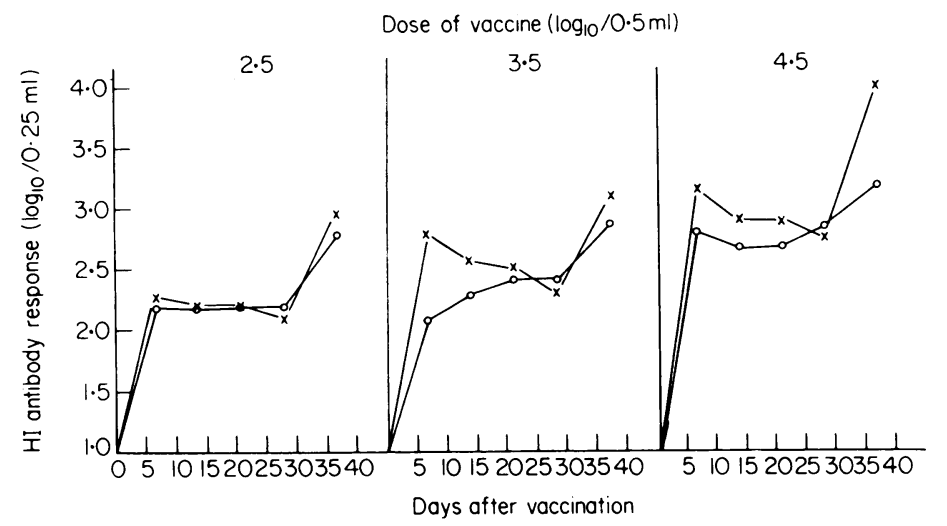

FIG. 2. Immune response of rabbits to A/Port Chalmers/73 intact influenza virus and subunit vaccines as measured in haemagglutination-inhibition (HI) tests.

Groups of three rabbits were injected with intact virus vaccine and haemagglutinin plus neuraminidase vaccines at equivalent doses. This subunit vaccine contained nucleocapsids. Values represent the mean HI titres per $0.25 \mathrm{ml}$ of serum and are expressed as the reciprocal of the dilution inhibiting three out of four haemagglutinin doses of virus. $X-X$, Intact virus vaccine; $\bigcirc-\bigcirc$ Subunit vaccine. 
73 virus were injected into companion rabbits. The antibody responses to the vaccine containing ribonucleoprotein (RNP) is shown in Fig. 2.

At low doses of vaccine ( $316 \mathrm{HAu})$ those rabbits which received subunits gave the same $\mathrm{HI}$ antibody response as those receiving intact virus. At higher doses $(31,600 \mathrm{HAu})$ there was significantly less early antibody produced by the subunits than by the intact virus, but the peak of the primary IgG antibody response was the same for intact virus and subunit vaccines.

The intact virus vaccine and the subunit vaccine also induced similar levels of antibodies to neuraminidase.

However, it has been suggested that RNA and DNA act as adjuvants in induction of immunity (Cowan, 1973) and it was therefore important to find whether the subunit vaccine had altered immunogenicity after removal of nucleocapsids.

The immunogenicity of the haemagglutinin and neuraminidase subunits (both with and without RNP antigen) was compared with that of intact A/Port Chalmers/ 73 virus as described above. It was found that removal of the RNP antigen from the subunit vaccine did not alter the immunogenicity of the HA and NA subunits. Addition of RNP to the HA plus NA vaccine did not change the immunogenicity of the subunits.

Immunogenicity in man of the haemagglutinin and neuraminidase subunits

Adult volunteers were injected with the Port Chalmers/73 haemagglutinin plus neuraminidase subunit vaccine and equivalent doses of intact, inactivated Port Chalmers virus.

The antibody responses of these people (Table 1) showed two things. First, the response to the subunits was as high as the response to the intact virus at equivalent concentration and second, the titres of the sera to the original member of the Hong Kong
$\left(\mathrm{H}_{3} \mathrm{~N}_{2}\right)$ series (A/Hong Kong/68) and the virus in $-\frac{2}{2}$ jected (A/Port Chalmers/74) were the same (Table 1).

Diverse response to 'specific' and 'common' antigenic $\stackrel{c}{\Rightarrow}$ determinants on the haemagglutinin subunits

Previous studies (Laver, Downie and Webster,을 1974; Virelizier, Allison and Schild, 1974) have shown을 that the haemagglutinin subunits of influenza viruses $\frac{\bar{c}}{\triangle}$ possess at least two different antigenic determinants. $\triangle$ Thus, the haemagglutinin subunits of influenzan viruses within a major strain (e.g. $\mathrm{H}_{3}$ ) possess $\mathrm{a}_{-}^{\mathrm{S}}$ common antigenic determinant(s) and variants with- in the strain (e.g. A/Hong Kong/68, A/Port Chal- $\vec{\omega}$ mers/73) possess one or more 'specific' antigenico determinants as well as the common antigenico determinant(s).

In double-immunodiffusion tests (Fig. 3) sera fromic rabbits vaccinated with Port Chalmers/73 haem-is agglutinin subunits showed clear-cut differences $\infty$ (spurs) between Port Chalmers/73 and Hong Kong/w 68 haemagglutinin subunits. These animals produced $\omega$ high levels of antibodies to the 'specific' determinants음 on Port Chalmers/73 haemagglutinin and low levelsof antibodies to the 'common' determinant(s). On ${ }_{\subseteq}^{\complement}$ the other hand, sera from adult humans vaccinated with Port Chalmers/73 virus contained antibodies $\overrightarrow{0}$ which gave a continuous line of precipitation tween the haemagglutinin subunits of Port Chalmes/? 73 and Hong Kong/68 influenza viruses (Fig. Thus, in contrast to the rabbits, these people re-s sponded only to the 'common' or 'cross-reacting' antigenic determinant on Port Chalmers/73 haem-气 agglutinin and did not respond at all to the 'specific' determinant(s).

These findings were confirmed in absorption ex- 3 periments. Absorption of rabbit antisera to Port? Chalmers/73 haemagglutinin with particles of Hong? Kong/68 virus removed all of the antibody reactingo with the Hong Kong/68 strain but left behind high levels of antibody reacting with Port Chalmers/73을

TABLE 1. Antibody response of humans to A/Port Chalmers/73 intact virus and HA + NA subunit vaccines*

\begin{tabular}{|c|c|c|c|c|c|}
\hline \multirow[b]{3}{*}{ Vaccine } & & \multicolumn{4}{|c|}{ HI response to the following influenza viruses } \\
\hline & & \multicolumn{2}{|c|}{ A/Port Chalmers/1/73 } & \multicolumn{2}{|c|}{ A/Hong Kong/68 } \\
\hline & & Prebleed & Primary & Prebleed & Primary \\
\hline Whole virus & (700 CCA u.) & $<$ & 2220 & 172 & 2341 \\
\hline $\mathrm{HA}+\mathrm{NA}$ subunits & $\begin{array}{l}\text { (Equivalent to } \\
700 \mathrm{CCA} \text { u. of virus) }\end{array}$ & $<$ & 5600 & 172 & 4530 \\
\hline $\mathrm{HA}+$ NA subunits & $\begin{array}{l}\text { (Equivalent to } \\
1400 \mathrm{CCA} \text { u. of virus) }\end{array}$ & $<$ & 3330 & 366 & 3083 \\
\hline
\end{tabular}

* Groups of 10 volunteers were iniected with the doses of vaccines shown. Values given are the average titres of the 10 sera.

$+<=$ Less than 20.

$\ddagger A$ recombinant virus $A / H o n g ~ K o n g / 68$ (NA)-A/BEL/42(HA) was used in these tests. 


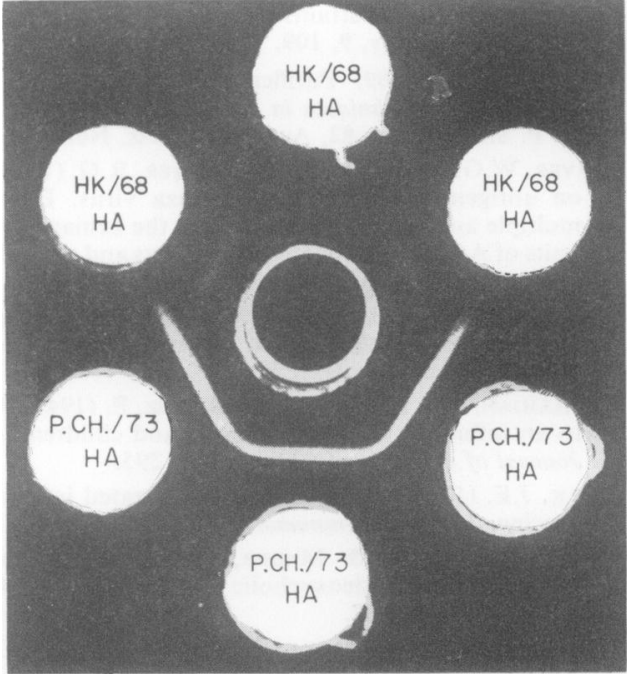

Rabbit serum in centre

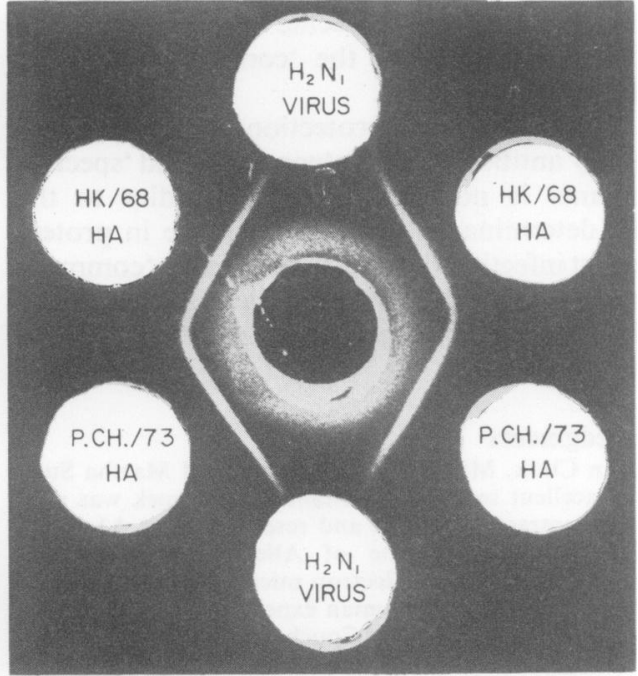

Human serum in centre

FIG. 3. Double immunodiffusion tests showing diversity in the immune response of rabbits and humans to A/Port Chalmers/73 haemagglutinin.

The rabbit has responded mainly to the 'specific' antigenic determinant(s) on Port Chalmers/73 haemagglutinin and the serum (left hand side) contains antibodies which react preferentially with this virus. The human, on the other hand. has responded exclusively to the 'common' antigenic determinant on Port Chalmers/73 haemagglutinin and the serum contains antibodies which react equally well with Hong Kong/68 and Port Chalmers/73 viruses. Absorption of the human serum with particles of Hong Kong/68 virus confirmed that no antibodies to the 'specific' determinants on Port Chalmers/73 haemagglutinin were present. No reaction with $\mathrm{H}_{2} \mathrm{~N}_{2}$ virus was obtained, showing that the human serum did not contain antibodies to the RNP or $\mathbf{M}$ protein antigens.

virus. Further absorption with Hong Kong/68 virus failed to remove any more antibody, indicating that the residual activity was directed against the 'specific' determinant(s) on Port Chalmers/73 haemagglutinin. (Absorption of the sera with Port Chalmers/73 virus totally removed all antibodies.)

In contrast, when sera from some people vaccinated with Port Chalmers/73 haemagglutinin (e.g. the serum shown in Fig. 3) were absorbed with Hong Kong/68 virus, all antibodies were totally removed. These sera contained antibodies which reacted only with the 'common' or 'cross-reacting' determinants on the haemagglutinin subunits of the two viruses and no antibodies reacting with the 'specific' determinant(s) on Port Chalmers/73 haemagglutinin.

A small number of the human sera did contain antibodies to the 'specific' determinant(s) on Port Chalmers/73 haemagglutinin (as well as to the 'common' determinant(s)) and, surprisingly, in some cases, vaccination of man with Port Chalmers/73 haemagglutinin induced antibodies reacting with the 'specific' determinant on Hong Kong/68 influenza virus haemagglutinin subunits (which is apparently absent in Port Chalmers/73 haemagglutinin).
Sera from people convalescent after a known infection with $\mathrm{A} /$ Port Chalmers/73 virus behaved in a similar way. Some contained antibodies directed only to the 'common' determinant(s) on the haemagglutinin subunits, while others contained antibodies to both 'common' and 'specific' determinants.

The volunteers in these experiments were chosen for their low levels of pre-vaccination antibodies to the Port Chalmers/73 and Hong Kong/68 influenza viruses. Nevertheless, it is likely that the volunteers had at some time experienced infection with one of the Hong Kong $\left(\mathrm{H}_{3} \mathrm{~N}_{2}\right)$ series of viruses and possessed cells 'primed' to $\mathrm{H}_{3}$ and $\mathrm{N}_{2}$ antigens.

This recapitulation of the immune response by a cross-reacting antigen to an antigen previously experienced is termed 'original antigenic sin' and has been well documented with many viral and non-viral antigens. In a small number of people, vaccinated with Port Chalmers/ 73 haemagglutinin, the sera contained antibodies directed against the 'specific' determinant on the Hong Kong/68 strain. This paradoxical antibody response has also recently been described by Virelizier et al. (1974).

A surprising finding was the complete absence of any antibodies to the 'specific' determinant on the Port Chalmers/73 haemagglutinin in many of the 
sera from people vaccinated with this antigen. In unprimed animals, the 'specific' determinant was more immunogenic than the 'common' or crossreacting determinant.

The relative degrees of protection against infection afforded by antibodies to the 'common' and 'specific' determinants is not known. If antibodies to the 'specific' determinants are more effective in protection against infection than antibodies to the 'common' determinant, then abrogation of the response to the 'specific' determinants on vaccines may render these ineffective.

\section{Acknowledgments}

Mrs Jean Clark, Miss Donna Cameron and Martha Sugg provided excellent technical assistance. This work was supported by Contract A1 42510 and research grant A1 08831 from the National Institute of Allergy and Infectious Diseases and by ALSAC. Electron micrographs were taken by $\operatorname{Dr}$ N. G. Wrigley. The human experiments were done in collaboration with Dr R. B. Couch and Dr J. A. Kasel, Baylor College of Medicine, Houston.

\section{References}

Cowan, K.M. (1973) Antibody response to viral antigens. Advances in Immunology, 17, 195.
LAVER, W.G. (1964) Structural studies on the protein subunits from three strains of influenza virus. Journal of Molecular Biology, 9, 109.

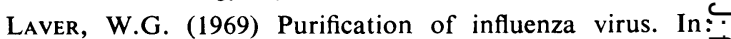
Fundamental Techniques in Virology (Ed. by K. Habel and N. P. Salzman), p. 82. Academic Press, New York.

Laver, W.G., Downie, J.C. \& Webster, R.G. (1974) Studie을 on antigenic variation in influenza virus. Evidence fo를 multiple antigenic determinants on the hemagglutinin sub $\overline{\bar{c}}$ units of $\mathrm{A} /$ Hong Kong/68 $\left(\mathrm{H}_{3} \mathrm{~N}_{2}\right)$ virus and the A/England/ 72 strains. Virology, 59, 230.

LAVER, W.G. \& VAlentine, R.C. (1969) Morphology of the isolated hemagglutinin and neuraminidase subunits of influenza virus. Virology, 38, 105.

Quilligan, J.J., Francis, T. \& Minuse, E. (1949) Reactions to an influenza vaccine in infants and children. Americaros Journal of Diseases of Children, 78, 295.

SALK, J.E. (1948) Reactions to concentrated influenza virus vaccine. Journal of Immunology, 58, 369.

Szalkowski, C.R. \& MADER, W.J. (1952) Colorometria determination of deoxycholic acid in ox bile. Analytica's Chemistry, 24, 1602.

Virelizier, J.L., Allison, A.C. \& Schild, G.C. (1974 Antibody responses to antigenic determinants of influenzaw virus hemagglutinin. II. Original antigenic sin: a bono marrow-derived lymphocyte memory phenomenon modu? lated by thymus-derived lymphocytes. Journal of Experimental Medicine, 140, 1571 . 